\title{
Acute adrenal cortex injury during cardiopulmonary bypass in a canine model
}

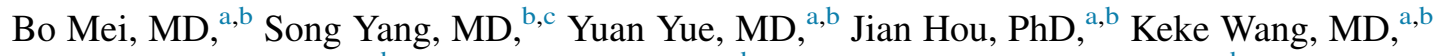
Guangxian Chen, MD, ${ }^{\mathrm{a}, \mathrm{b}}$ Mengya Liang, MD, ${ }^{\mathrm{a}, \mathrm{b}}$ and Zhongkai $\mathrm{Wu}, \mathrm{MD}, \mathrm{PhD}^{\mathrm{a}, \mathrm{b}}$

\section{ABSTRACT}

Objective: Cardiopulmonary bypass (CPB) might induce systemic inflammatory responses that cause acute injuries to multiple organs. However, no direct evidence exists to determine whether CPB leads to adrenal cortex injury or to describe its underlying mechanism.

Methods: Twelve healthy adult beagles were randomly assigned into control and CPB groups. After cannulation, mild hypothermia CPB was performed in the CPB group but not in the control group. The serum concentrations of various cytokines, cortisol, and aldosterone were assessed. Adrenal cortex injuries were evaluated using standard histological methods. Steroidogenic enzymes and the nucleotide-binding oligomerization domain-like receptor containing pyrin domain 3 (NLRP3) inflammasome pathway were detected using quantitative polymerase chain reaction and Western blot analysis.

Results: During CPB, serum interleukin (IL)-6, IL-8, IL-10, tumor necrosis factor $\alpha$, cortisol, and aldosterone levels were significantly higher in the CPB group. The pathologic study revealed higher injury scores $(3.6 \pm 0.6$ vs $0.7 \pm 0.7)$ and significantly more severe edema, inflammatory cell infiltration (lymphocytes and neutrophils), and apoptosis in the CPB group. The electron microscopic examination showed swollen mitochondria, ruptured mitochondrial cristae, reduced lipid droplets, and increased secondary lysosomes in the CPB group. The mRNA expression levels of NLRP3 and the protein levels of $17 \alpha$-hydroxylase and IL- $1 \beta$ in adrenal tissue were significantly upregulated in the CPB group.

Conclusions: CPB induces significant systemic and local inflammation in the adrenal cortex and results in cytological architectural and ultrastructural alterations in adrenocorticocytes. In addition, the NLRP3 inflammasome pathway might promote adrenal gland injury during CPB and might represent a novel potential therapeutic target. (J Thorac Cardiovasc Surg 2018;156:696-706)

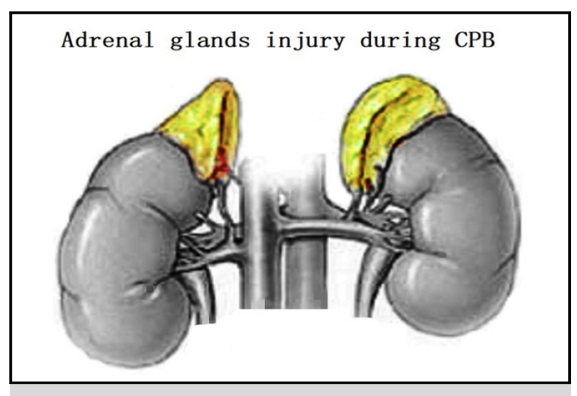

Acute adrenal cortex injury during cardiopulmonary bypass.

\section{Central Message}

$\mathrm{CPB}$ induces significant local inflammation in the adrenal cortex and results in cytological architectural and ultrastructural alterations. The NLRP3 inflammasome pathway might promote this injury.

\section{Perspective}

In the clinical setting, extensive disagreements over treatment outcomes and the necessity of exogenous prophylactic steroids during $\mathrm{CPB}$ are becoming increasingly prominent. Thus far, no direct evidence exists to determine whether $\mathrm{CPB}$ leads to adrenal injury during cardiac surgery. Therefore, it is necessary to determine whether the adrenal cortex is injured and how it becomes injured during $\mathrm{CPB}$.

See Editorial Commentary page 707.
Cardiopulmonary bypass (CPB) is used in most cardiac surgeries and in other diseases. CPB induces an inflammatory

\footnotetext{
From the Departments of ${ }^{\mathrm{a} C a r d i a c}$ Surgery, and ${ }^{\mathrm{c}}$ Cardiosurgery Intensive Care Unit, First Affiliated Hospital of Sun Yat-Sen University, Guangzhou, China; and ${ }^{\mathrm{b}} \mathrm{Key}$ Laboratory on Assisted Circulation, Ministry of Health, Guangzhou, China.

This work was supported by the National Natural Science Foundation of China (81370215, 81570039)

Drs Mei and Yang contributed equally to this work.

Received for publication Nov 9, 2017; revisions received March 4, 2018; accepted for publication March 5, 2018; available ahead of print May 9, 2018.

Address for reprints: Zhongkai Wu, MD, PhD, Department of Cardiac Surgery, First Affiliated Hospital of Sun Yat-Sen University, 58 Zhongshan II Rd, Guangzhou 510080, China (E-mail: wuzhk@mail.sysu.edu.cn).

$0022-5223 / \$ 36.00$

Copyright (c) 2018 by The American Association for Thoracic Surgery

https://doi.org/10.1016/j.jtcvs.2018.03.151
}

response and injury in patients in sensitive organs such as the heart, ${ }^{1}$ lungs, ${ }^{2}$ brain, ${ }^{3}$ kidneys, ${ }^{4}$ and intestines. ${ }^{5}$ However, no direct evidence exists to determine whether CPB leads to adrenal injury during cardiac surgery.

The adrenal cortex is responsible for producing important agents, including corticosteroids and mineralocorticoids. These substances play several critical roles in maintaining the normal function of the human body, especially during

- Scanning this $\mathrm{QR}$ code will take you to a supplemental video for the article. 


$$
\begin{aligned}
& \text { Abbreviations and Acronyms } \\
& \begin{aligned}
& \mathrm{CON}=\text { control } \\
& \mathrm{CPB}=\text { cardiopulmonary bypass } \\
& \mathrm{CYP} 17=17 \alpha \text {-hydroxylase } \\
& 3 \beta \mathrm{HSD}=3 \beta \text {-hydroxysteroid dehydrogenase } \\
& \mathrm{IL} \quad=\text { interleukin } \\
& \mathrm{I} / \mathrm{R} \quad=\text { ischemia-reperfusion } \\
& \mathrm{NLRP}= \text { nucleotide-binding oligomerization } \\
& \text { domain-like receptor containing pyrin } \\
& \text { domain } 3 \\
& \mathrm{TNF}= \text { tumor necrosis factor }
\end{aligned}
\end{aligned}
$$

cardiac surgery. Corticosteroids can suppress the inflammation induced by CPB. In the clinical setting, exogenous prophylactic steroids are used to strengthen the effect of inflammation suppression during $\mathrm{CPB}$ or to provide replacement therapy for adrenal insufficiency after CPB. However, extensive disagreements over treatment outcomes and the necessity of steroids are becoming increasingly prominent. ${ }^{1,6-11}$ Therefore, to guide clinical therapy, it might be helpful to determine whether the adrenal cortex is injured and how it becomes injured during CPB.

Interleukin (IL)- $1 \beta$ and IL-18 are quintessential proinflammatory cytokines that broadly affect inflammatory processes. $^{12}$ These cytokines play important roles in organ injury during CPB. ${ }^{13,14}$ The maturation of IL- $1 \beta$ and IL18 is dependent on nucleotide-binding oligomerization domain-like receptor containing pyrin domain 3 (NLRP3) inflammasome assembly and its subsequent activation. The NLRP3 inflammasome is also involved in injurious inflammatory responses in various diseases, including myocardial ischemia-reperfusion (I/R) injury, ${ }^{15,16}$ cardiomyocyte apoptosis, ${ }^{15}$ remodeling after myocardial infarct, ${ }^{16}$ alveolar barrier dysfunction, ${ }^{17}$ pulmonary artery remodeling in pulmonary hypertension, ${ }^{18}$ and intestinal inflammation. ${ }^{19}$ However, little is known about the NLRP3 inflammasome pathway in the adrenal glands during CPB.

On the basis of these considerations, we hypothesized that $\mathrm{CPB}$ injures the adrenal cortex, and activation of the NLRP3 inflammasome pathway is involved in CPBinduced adrenal injury.

\section{METHODS}

\section{Animals and Experimental Design}

All canine work was conducted under Sun Yat-sen University Institutional Animal Care and Use Committee guidelines. Twelve healthy adult beagles of either sex (6-8 years old, weight $14.2 \pm 1.5 \mathrm{~kg}$, Guangdong Qianyan Biological Technology Co Ltd, Guangzhou, China) were randomly assigned to 2 groups: the control $(\mathrm{CON})$ group $(n=6)$ and the CPB group $(n=6)$.

For the CPB group, the protocol for anesthesia and the establishment of CPB were described in detail previously (Video 1). ${ }^{20,21}$ In brief, after

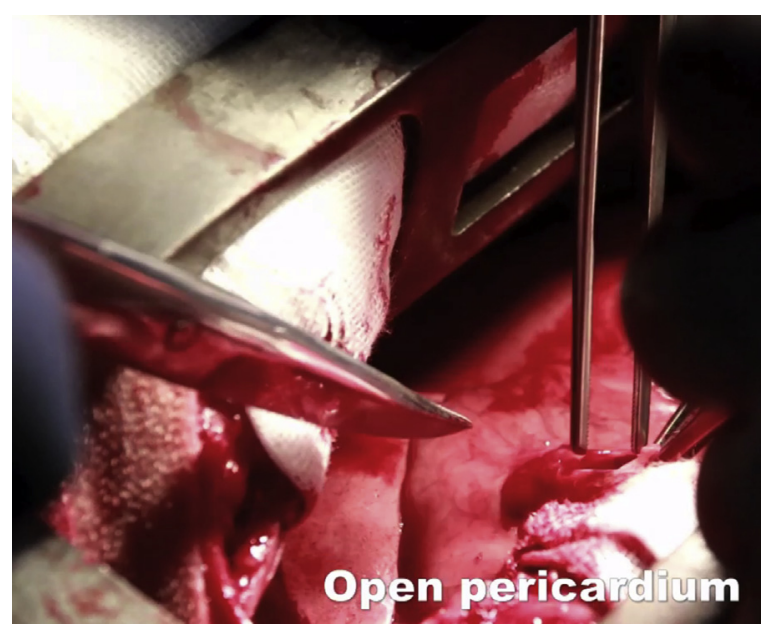

VIDEO 1. Acute adrenal cortex injury during cardiopulmonary bypass in a canine model. 1. Cardiopulmonary bypass may induce systemic inflammatory responses that cause acute injuries to multiple organs such as the heart, lungs, brain, kidneys, and intestines. The adrenal cortex produces corticosteroids which plays important roles in maintaining the normal function of the human body, especially during cardiac surgery. However, no direct evidence exists to determine whether CPB leads to adrenal cortex injury. 2. Twelve healthy adult beagles were randomly assigned into CON group and CPB group. 3. All beagles were weighed. 4. Ketamine, fentanyl, and vecuronium were used for anesthesia induction via ear marginal venous. Anesthesia was maintained with vecuronium, fentanyl, and midazolam. 5. After induction of anesthesia, oral intubations were performed Then animals were placed on a ventilator. 6. Skin preparation and disinfection were performed. Arterial and venous catheters were then placed into the femoral vein and artery to collect blood samples and monitor hemodynamic parameters. ECG was monitored. 7. Median thoracotomy was performed. Stay sutures were placed on the pericardium. 8. The CPB circuit and the pediatric membrane oxygenator were primed with solution and multiple electrolyte injections. 9. After heparinization, 16-French cannulas were introduced into the aorta, whereas an 18-French cannula was used in the right atrium and superior vena cava. 10. After CPB was established, cardiac arrest was induced by $4^{\circ} \mathrm{C}$ crystalloid cardioplegia, and the animal underwent 60 minutes of cardiac arrest. During CPB, the flow rate was maintained between 50 and $80 \mathrm{~mL} / \mathrm{kg} / \mathrm{min}$, the mean arterial pressure was between 50 and $80 \mathrm{~mm} \mathrm{Hg}$, and the temperature was maintained at $36^{\circ} \mathrm{C}$. 11 . After 60 minutes of cardiac arrest the aortic clamp was removed and the heart beat restored spontaneously. 12. After 60 minutes of reperfusion. The beagles were euthanized, the blood samples were collected and a laparotomy was performed, and then the adrenal glands were fetched. The adrenal glands were divided into several parts. The adrenal cortex was dissected carefully from one specimen and placed in liquid nitrogen immediately for molecular testing. One specimen was fixed in $10 \%$ formaldehyde for light microscopy. Another specimen was immersed in $2.5 \%$ glutaraldehyde for electron microscopy examination. 13. At last, the incision was closed after collecting samples. Video available at: https://www.jtcvs.org/article/S0022-5223(18)30944-9/fulltext.

anesthesia induction (ketamine $30 \mathrm{mg} / \mathrm{kg}$; fentanyl citrate $2 \mu \mathrm{g} / \mathrm{kg}$; vecuronium bromide $0.1 \mathrm{mg} / \mathrm{kg}$ ), tracheal intubation was performed, and the animals were placed on a ventilator (BIRD VELA Model 606A; Carefusion, San Diego, Calif). Anesthesia was maintained with an intravenous infusion of vecuronium bromide, fentanyl, and midazolam. Central venous catheters and fluid-filled catheters were then placed into 

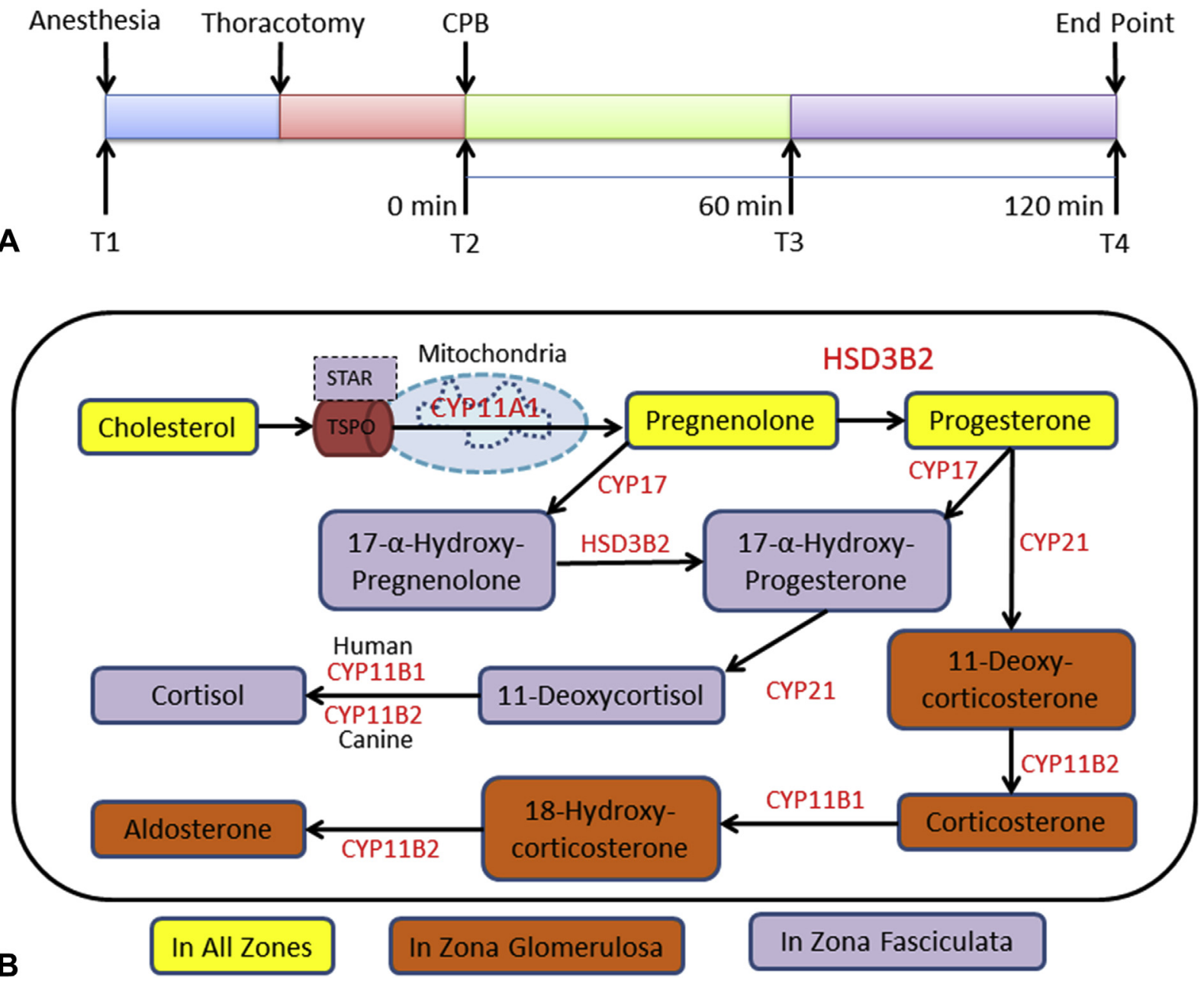

FIGURE 1. Experimental protocol (A) and the cortisol and aldosterone steroidogenesis pathways in humans and canines (B). $C P B$, Cardiopulmonary bypass; StAR, steroidogenic acute regulatory protein; TSPO, translocator proteins; CYP11A1, cytochrome P450 side chain cleavage; HSD3B2, 3 $\beta$ hydroxysteroid dehydrogenase type II; $C Y P 17,17 \alpha$-hydroxylase; $C Y P 21,21$-hydroxylase; $C Y P 11 B 1$, 11 $\beta$-hydroxylase; $C Y P 11 B 2$, aldosterone synthase.

the left femoral vein and femoral artery to collect blood samples and monitor hemodynamic parameters (MP150; BIOPAC Systems Inc, Camino Goleta, Calif), respectively.

After median sternotomy, CPB was performed using one roller pump for extracorporeal circulation and another roller pump for suction. The extracorporeal circuit and the pediatric membrane oxygenator (Maquet 31000; Maquet Co, Hirrlingen, Germany) were primed with solution (average $568 \pm 73.0 \mathrm{~mL}$ ) and multiple electrolyte injections. After heparinization (heparin $300 \mathrm{U} / \mathrm{kg}$ ), a 16-French cannula (Edwards Lifesciences LLC, Irvine, Calif) was introduced into the aorta, whereas an 18-French cannula was introduced into the right atrium and superior vena cava. Then, cardiac arrest was induced with homemade $4{ }^{\circ} \mathrm{C}$ crystalloid cardioplegia, ${ }^{21}$ and the canine underwent 60 minutes of aortic clamping. During CPB, the activated clotting time was maintained longer than 480 seconds. The flow rate was maintained between 50 and $80 \mathrm{~mL} / \mathrm{kg} / \mathrm{min}$, the mean arterial pressure was between 50 and $80 \mathrm{~mm} \mathrm{Hg}$, and the nasopharyngeal temperature $\left(36^{\circ} \mathrm{C}\right)$ was maintained using a heat exchanger (Sarns Heater/Cooler, Ann Arbor, Mich). In the CON group, after heparinization (heparin $300 \mathrm{U} / \mathrm{kg}$ ), aortic, superior vena cava, and right atrium cannulations were established without CPB. Canines were euthanatized after 60 minutes of reperfusion in the $\mathrm{CPB}$ group, and 120 minutes after aorta and right atrium cannulation in the CON group (Figure 1, $A$ ). A laparotomy was performed, and the adrenal glands were harvested and placed on ice immediately, after which they were divided into 2 parts. One specimen was fixed in $10 \%$ formaldehyde for light microscopy. The adrenal cortex was dissected carefully from another specimen. A small part of the cortex (approximately $1 \mathrm{~mm}^{3}$ ) was immersed in $2.5 \%$ glutaraldehyde for electron microscopy examination and the rest of the specimen was immediately placed in liquid nitrogen for molecular biological detection. Blood samples from the femoral artery were collected at designated time points (CPB group: T1, after anesthesia; $\mathrm{T} 2$, immediately after establishment of $\mathrm{CPB}$ cannulation; T3, 60 minutes after $\mathrm{CPB}$ cannulation; and $\mathrm{T} 4,120$ minutes after $\mathrm{CPB}$ cannulation; Figure 1, A).

\section{Enzyme-Linked Immunosorbent Assay}

Blood samples were collected in prechilled ethylene diamine tetraacetic acid tubes at designated time points as previously described, placed on ice, and centrifuged at $4{ }^{\circ} \mathrm{C}$, and the plasma was stored at $-80^{\circ} \mathrm{C}$ until analysis. The total levels of IL-6, 8, 10, tumor necrosis factor (TNF)- $\alpha$, cortisol, and aldosterone were analyzed using a canine-specific enzyme-linked immunosorbent assay kit according to the manufacturer's instructions using an Immulite 2000 (Diagnostic Products Corp, Los Angeles, Calif). The serum concentrations of IL-6, 8, 10, TNF- $\alpha$, cortisol, and aldosterone were 
TABLE 1. Adrenal histopathologic scoring criteria

\begin{tabular}{ll}
\hline Score & Edema \\
\hline & Absent \\
\hline 0 & $\leq 25 \%$ edema cells per HPF \\
\hline 1 & $>25 \%$ and $\leq 50 \%$ edema cells per HPF \\
2 & $>50 \%$ and $\leq 75 \%$ edema cells per HPF \\
\hline 3 & $>75 \%$ edema cells per HPF \\
\hline 4 & Leukocyte infiltration \\
\hline 0 & Absent \\
\hline 1 & 1 to 5 infiltrated cells per HPF \\
\hline 2 & 6 to 10 infiltrated cells per HPF \\
\hline 3 & 11 to 15 infiltrated cells per HPF \\
\hline 4 & $\geq 16$ infiltrated cells per HPF \\
\hline 0 & Necrosis and apoptosis \\
\hline 1 & Absent \\
\hline 2 & 1 to 5 necrotic cells per HPF \\
\hline 3 & 5 to 10 necrotic cells per HPF \\
\hline 4 & 11 to 15 necrotic cells per HPF \\
\hline$H P F, ~$ & $\geq 16$ necrotic cells per HPF \\
\hline
\end{tabular}

$H P F$, High-power field.

assessed using specific kits (IL-6, 8, 10, TNF- $\alpha$, and cortisol: product CSB-E11260c, CSB-E08052c, CSB-E11261c, CSB-E11737c, and CSB-E14303c, respectively. Cusabio Biotech Co, Ltd, Wuhan, China; Aldosterone: product CEA911Ge, Cloud-Clone Corp Houston, Tex).

\section{Histopathological Analysis}

Sectioning and hematoxylin and eosin staining were conducted according to standard histological methods. Two pathologists evaluated all sections independently. The histopathologic evaluation of the adrenal gland was conducted using a standardized scoring method (Table 1). The average of the scores (10 fields in each section) was recorded as the final histopathologic score.

\section{Fluorescent Immunohistochemistry}

A fluorescent immunohistochemistry assay was performed on $4-\mu \mathrm{m}$ tissue sections. Paraffin slides were deparaffinized and rehydrated. Antigen retrieval was achieved by incubating the sections at $37^{\circ} \mathrm{C}$ for 25 minutes. The sections were permeabilized in $0.2 \%$ Triton X-100 (product G1204, Servicebio, Wuhan Servicebio Technology Co, LTD, Wuhan, China) for 20 minutes at room temperature, and then incubated with a TUNEL kit (product 11684817910, Roche) for 2 hours at room temperature. After a final series of rinses, nuclei were stained with DAPI (product G1012, Servicebio) for 10 minutes at room temperature in the dark. Immunolabeled tissues were visualized with epifluorescence microscopy (Nikon Eclipse CI; Nikon Instruments Inc, Melville, NY).

\section{Electron Microscopy}

Another specimen was immersed in $2.5 \%$ glutaraldehyde and then postfixed in $2 \%$ osmium tetroxide in sodium phosphate buffer for 2 hours at $4^{\circ} \mathrm{C}$. The samples were dehydrated in a graded series of ethanol and propylene oxide. Then, all samples were embedded in araldite. One-micrometer sections were cut with an Ultramicrotome (Leica EM UC7; Leica, Nussloch, Germany). After staining with lead citrate and uranyl acetate, the sections were observed using a Hitachi Transmission Electron Microscope (HT7700; Hitachi, Tokyo, Japan).

\section{Real-Time Polymerase Chain Reaction}

Total mRNA was extracted from adrenal cortex specimens with the TRIzol reagent (Invitrogen). RNA was quantified using a Thermo Scientific Nanodrop 2000. Total mRNA $(2 \mu \mathrm{g})$ was reverse-transcribed using random hexamers. Complementary DNA levels of steroidogenic enzymes (steroidogenic acute regulatory protein), cytochrome $\mathrm{P} 450$ side chain cleavage, $17 \alpha$-hydroxylase (CYP17), $3 \beta$-hydroxysteroid dehydrogenase $(3 \beta \mathrm{HSD}), 21$-hydroxylase, and aldosterone synthase, NLRP3, caspase-1, IL-1 $\beta$, and IL-18 were quantified in real time using the StepOnePlus System (Applied Biosystems, Carlsbad, Calif; Figure 1,B). The data are expressed relative to glyceraldehyde-3phosphate dehydrogenase expression. All target gene primer sequences were designed on the basis of the NCBI GenBank database (http:// www.ncbi.nlm.nih.gov) and are listed in Table 2. All samples were assayed in triplicate. The delta-delta $\mathrm{Ct}$ method was used to calculate the relative mRNA expression levels.

\section{Western Blot Analysis}

Frozen samples of adrenal cortex tissue were homogenized in RIPA buffer. The primary antibodies used for the Western blot analysis were anti-CYP17 antibody (ab48019, Cambridge, United Kingdom), anti-3 $\beta$ HSD antibody (ab80500), anti-IL-1 $\beta$ antibody (ab106015), and anti-NLRP3 antibody (NBP1-52154, Novus Biologicals, Colo). Five micrograms of total protein was subjected to $5 \%$ polyacrylamide gel electrophoresis and blotted onto polyvinylidene difluoride membranes (Merck Millipore, Germany). The membranes were blocked for 1 hour in 5\% nonfat milk in Tris-buffered saline with Tween-20 at room temperature, and then incubated overnight at $4{ }^{\circ} \mathrm{C}$ with anti-3 $\beta \mathrm{HSD}$ antibody in a 1:500 dilution, anti-CYP17 antibody in a 1:1000 dilution, anti-IL-1 $\beta$ antibody in a 1:1000 dilution, and anti-NLRP3 antibody in a $1: 1000$ dilution diluted in $0.5 \%$ Tris-buffered saline with Tween-20 in $5 \%$ bovine serum albumin. Then, the membranes were incubated for 30 minutes at room temperature with the secondary antibody (anti-rabbit, horseradish peroxidase conjugated, 1:3000). Protein bands were visualized with an electrogenerated chemiluminescence advanced Western blot detection kit on X-ray film (Kodak, Rochester, NY). $\beta$-actin (product GB13001-1, Wuhan Servicebio Technology Co) was used as a loading control.

\section{Statistical Analysis}

All statistical analyses were performed using IBM SPSS Statistics version 22.0 (IBM Corp, Armonk, NY). Parametric data are presented as mean \pm standard error of the mean. Intergroup mean comparisons of parametric data were performed with the independent-samples $t$ test. The statistical analysis of blood biochemical indexes was performed using a repeated measures analysis of variance. A $P$ value $<.05$ was considered statistically significant.

\section{RESULTS}

All canines survived the surgical procedures. The blood biochemical indexes of the canines during the procedure are summarized in Figure 2. In the CPB group, the serum concentrations of IL-6, IL-8, IL-10, TNF- $\alpha$, and cortisol increased gradually during the procedure, and the serum aldosterone concentration tended to increase. Compared with the CON group, the serum cortisol concentration and the serum aldosterone concentration were significantly increased at $\mathrm{T} 3$ and $\mathrm{T} 4$ in the $\mathrm{CPB}$ group $(P<.05)$. 
TABLE 2. List of the primers used for PCR of various genes in adrenal glands

\begin{tabular}{|c|c|c|c|c|}
\hline Target gene & & Sequence $\left(5^{\prime} \rightarrow 3^{\prime}\right)$ & Product size, bp & Accession number \\
\hline \multirow[t]{2}{*}{ StAR } & For & CTCTGCTTGGTTCTCGG & 125 & NM_001097542.1 \\
\hline & $\operatorname{Rev}$ & СCTTCTTCCAGCCTTCC & & \\
\hline \multirow[t]{2}{*}{ CYP11A1 } & For & CACCGCCTCCTTAAAAAGTAACAAG & 129 & XM_535539.3 \\
\hline & $\operatorname{Rev}$ & GCTGCGTGCCATCTCGTAG & & \\
\hline \multirow[t]{2}{*}{ CYP17 } & For & CCTGCGGCCCCTATGCTC & 134 & XM_535000.3 \\
\hline & $\operatorname{Rev}$ & GGCCGGTACCACTCCTTCTCA & & \\
\hline \multirow[t]{2}{*}{$3 \beta H S D$} & For & CAGGAGGGTTTCTGGGTCAG & 186 & NM_001010954 \\
\hline & Rev & AGGCTCTCTTCAGGCACTGC & & \\
\hline \multirow[t]{2}{*}{ CYP21 } & For & AGCCCGACCTTCCCCTCCACCTG & 152 & NM_001003335 \\
\hline & $\operatorname{Rev}$ & TCTGCCGGCGAAGTCCACCCATTT & & \\
\hline \multirow[t]{2}{*}{$C Y P 11 B 2$} & For & GCCTACCCCTTGTGGATGAC & 126 & XM_00343180587.1 \\
\hline & Rev & CTCTGTGACTGCTGTCTGGG & & \\
\hline \multirow[t]{2}{*}{ NLRP3 } & For & СCTCATACCACCTATGCTTATCG & 169 & XM_843284.4 \\
\hline & $\operatorname{Rev}$ & GCTGGAGCATTTCACATAACAC & & \\
\hline \multirow[t]{2}{*}{ Caspase-1 } & For & TGAGGACATTCACCTGGCACA & 115 & NM_001003125.1 \\
\hline & Rev & TTCCACGAAGGCTGGTAAAGA & & \\
\hline \multirow[t]{2}{*}{$I L-1 \beta$} & For & CAAGAGTCTGAGGCATTTCGTG & 220 & NM_001037971.1 \\
\hline & Rev & CACCAGGTATTTGTGGCTTATGTC & & \\
\hline \multirow[t]{2}{*}{$I L-18$} & For & ATGGCTGCTAACCTAATAGAAGAC & 96 & NM_001003169.1 \\
\hline & Rev & GCCTTCATCACTTTCCGCTTT & & \\
\hline \multirow[t]{2}{*}{$G A P D H$} & For & GGGTGATGCTGGTGCTGAGTAT & 143 & NM_017008 \\
\hline & Rev & TTGCTGACAATCTTGAGGGAGTT & & \\
\hline
\end{tabular}

$\overline{b p}$, Base pairs; StAR, steroidogenic acute regulatory protein; For, forward primer; Rev, reverse primer; $C Y P 11 A 1$, cytochrome P450 side chain cleavage; $C Y P 17,17 \alpha$-hydroxylase; $3 \beta H S D, 3 \beta$-hydroxysteroid dehydrogenase; $C Y P 21$, 21-hydroxylase; $C Y P 11 B 2$, aldosterone synthase; $N L R P 3$, nucleotide-binding oligomerization domain-like receptor containing pyrin domain 3; $I L$, interleukin; $G A P D H$, glyceraldehyde-3-phosphate dehydrogenase.

\section{Histopathological Injury Scores}

The extent of adrenal cortex damage in both groups, which was assessed using histologic analyses and TUNEL assays, is shown in Figure 3. Histopathological injury scores were on the basis of edema, leukocyte infiltration, hemorrhage, necrosis, and apoptosis. The scores were significantly higher in the CPB group than those in the CON group $(P<.05)$. Significant differences were observed in the CPB group for edema, inflammatory cell infiltration, and apoptosis.

\section{Electron Microscopy}

The electron microscopic analysis of adrenal cells is shown in Figure 4. The ultrastructures of the fascicular and glomerulosa zona cells showed no pathological alterations in the CON group. Mitochondrial injury was more serious in the fascicular and glomerulosa zona cells in the $\mathrm{CPB}$ group than that in the CON group. In the CPB group, the major alterations in the mitochondria included edema and rupture of the mitochondrial cristae. Additionally, a reduction in lipid droplets was obvious. Moreover, a notable increase in secondary lysosomes was observed.

\section{Steroidogenic Enzymes}

Real-time polymerase chain reaction showed that the relative mRNA levels of steroidogenic enzymes were not different in the 2 groups (Figure 5, A). The Western blot analysis of CYP17 and $3 \beta \mathrm{HSD}$ in adrenal tissue is shown (Figure 5, $B$ and $C$ ). No significant difference was found in $3 \beta \mathrm{HSD}$ expression between the 2 groups. However, CYP17 expression was significantly greater in the CPB group than that in the CON group $(P<.05)$.

\section{The NLRP3 Inflammasome Pathway}

The relative mRNA levels of NLRP3 were significantly higher in the $\mathrm{CPB}$ group than those in the CON group $(P<.05)$, but caspase-1, IL-1 $\beta$, and IL-18 were not different in the 2 groups (Figure $6, A$ ). The Western blot analysis of NLRP3 and IL-1 $\beta$ in adrenal tissue is shown in Figure 6, $B$ and $C$. NLRP3 and IL- $1 \beta$ were significantly upregulated in the CPB group.

\section{DISCUSSION}

In the current study, we show that CPB induces significant systemic and local inflammation in the adrenal tissue. CPB 

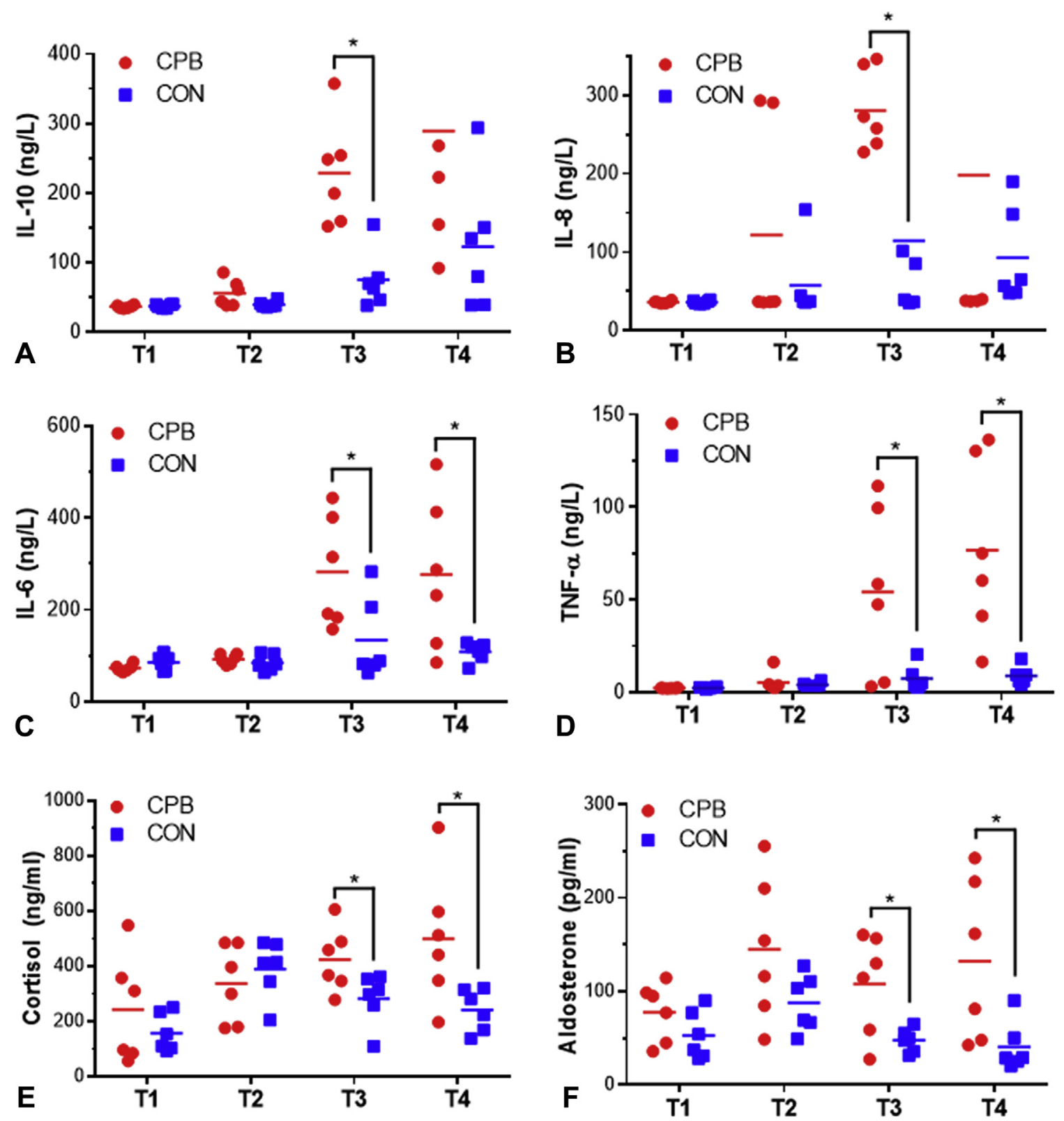

FIGURE 2. The serum concentrations of differential cytokines, cortisol, and aldosterone (compared with the control $[C O N]$ group, $* P<.05)$. A, Interleukin (IL)-10; B, IL-8; C, IL-6; D, TNF- $\alpha$; E, cortisol; and F, aldosterone. CPB, Cardiopulmonary bypass; $T 1$ to $T 4$, time 1 through 4; TNF, tumor necrosis factor.

can induce cytokine release, including IL-6, IL-8, IL-10, and $\mathrm{TNF}-\alpha$, and these cytokines might result in CPB-induced organ injury, which is consistent with previous reports. ${ }^{1-5}$ The canines exhibited immediate endocrine stress and inflammatory responses. The serum cortisol concentration increased gradually during CPB. Furthermore, CPB accelerates the synthesis and expression of steroidogenic key enzymes (CYP17) in adrenal tissue. Overall, the findings were consistent with those of human patients who exhibit distinct intraoperative increases in cortisol production elicited by CPB. ${ }^{22,23}$
The adrenal cortex is responsible for producing corticosteroids and mineralocorticoids. Corticosteroids affect the metabolism of sugar, fat, and protein, suppress the inflammation induced by $\mathrm{CPB}$, and enhance the sensitivity of vascular smooth muscle to catecholamine, reducing the permeability of capillaries and helping to maintain blood volume during intraoperative and postoperative periods. Aldosterone, secreted by the adrenal cortex, plays important roles in cardiovascular regulation and electrolyte metabolism. ${ }^{24}$ Hence, hormonal fluctuations caused by injured adrenal glands might affect the outcomes in cardiac surgery. 

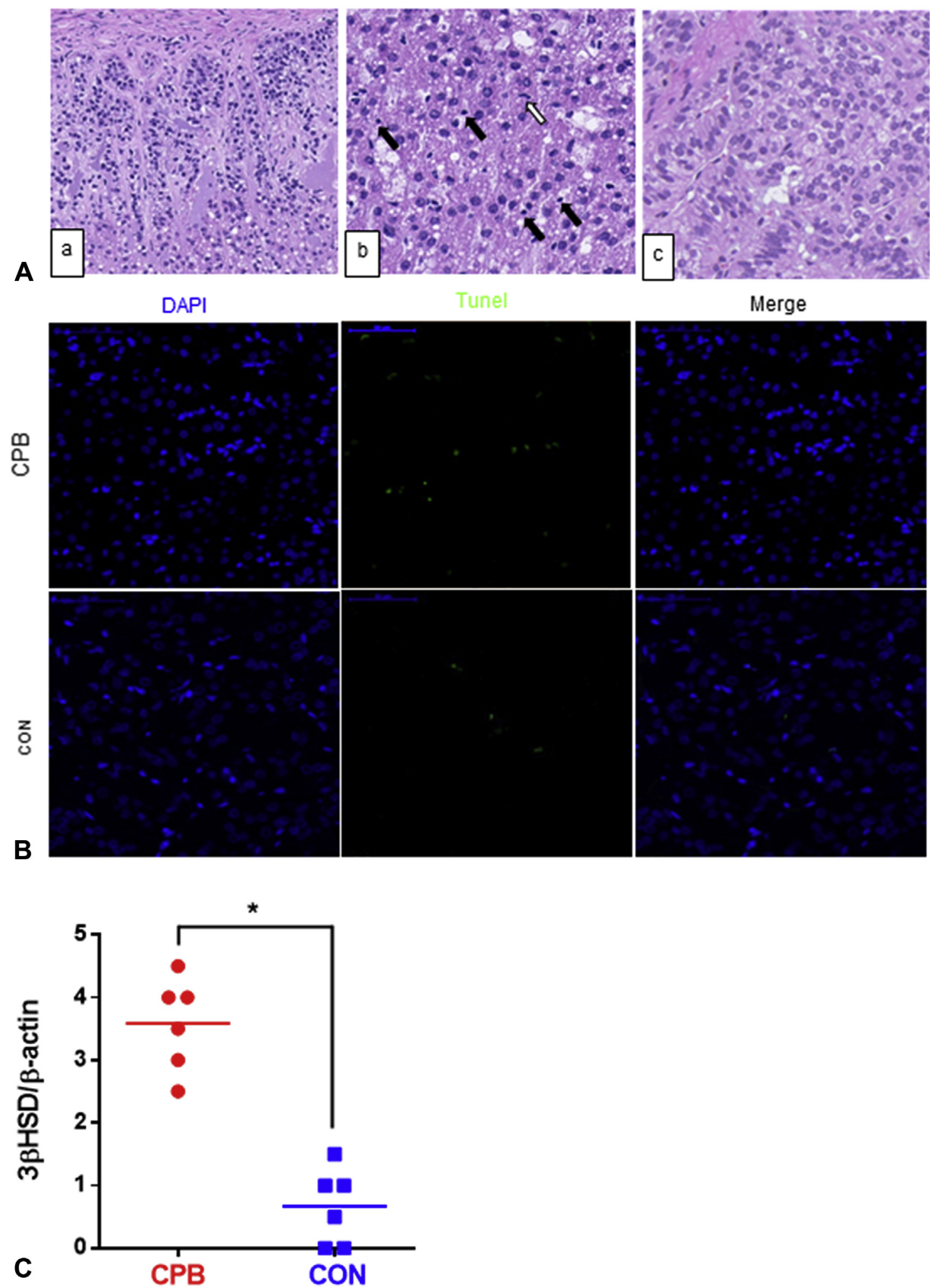

FIGURE 3. Assessment of adrenal injury using hematoxylin and eosin staining (A) and TUNEL assay (B) of adrenal gland sections. The expression of cell edema $(a)$ and inflammatory cell infiltration in the cardiopulmonary bypass $(C P B)$ group $(b)$; no structural alterations in the control $(C O N)$ group $(c)$. B, The expression of apoptosis. C, Adrenal histopathological scores (solid arrow: lymphocytes; open arrow: neutrophils; original magnification: 400 $\times$ ). $D A P I, 4^{\prime}$,6-diamidino-2-phenylindole; $3 \beta H S D, 3 \beta$-hydroxysteroid dehydrogenase. 

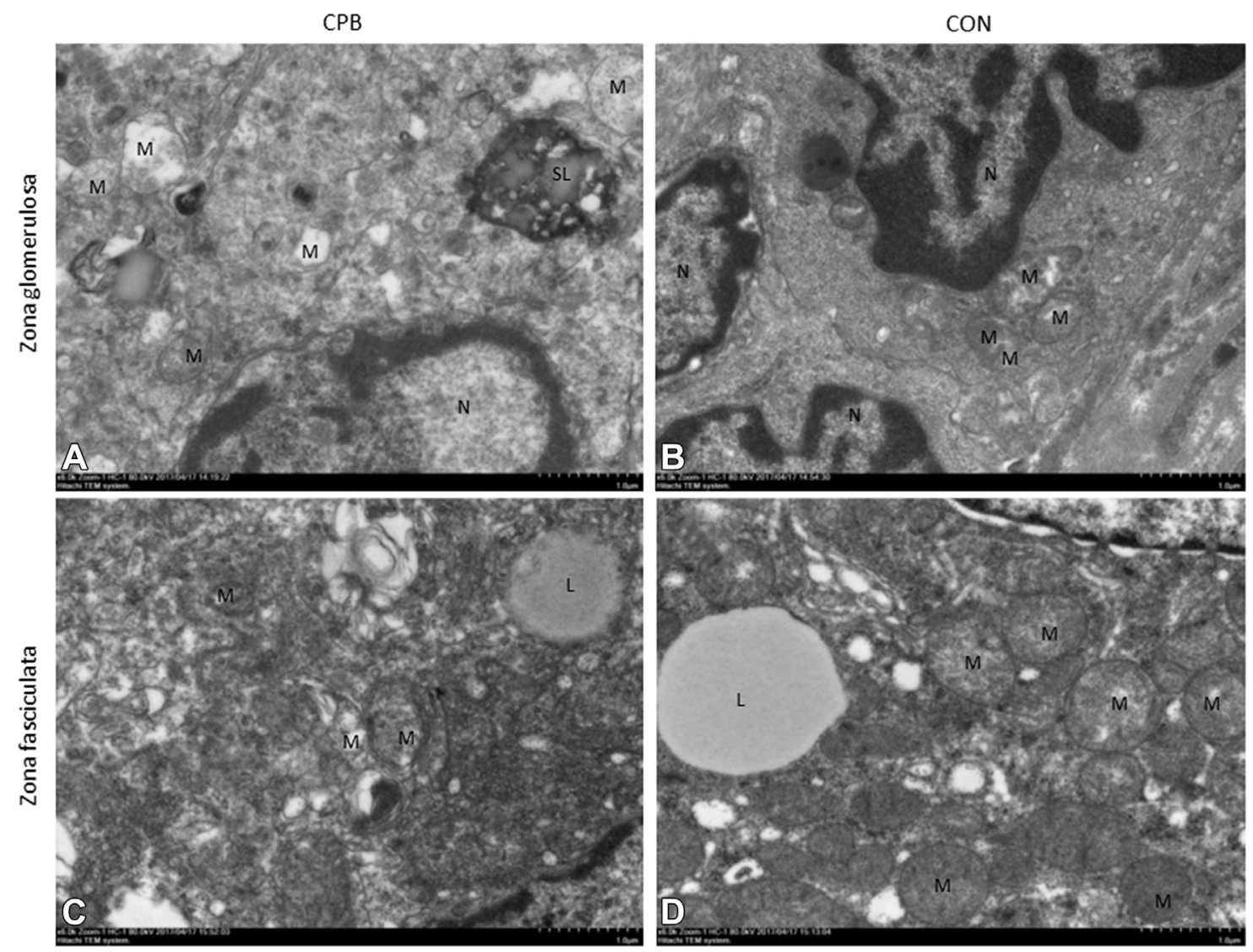

FIGURE 4. Electron microscopic analysis of adrenal cells. A and B, Cardiopulmonary bypass $(C P B)$-induced swollen mitochondria, ruptured mitochondrial cristae, reduced lipid droplets, and increased secondary lysosomes. $\mathrm{C}$ and $\mathrm{D}$, The ultrastructures of adrenal cortex cells showed no alterations in the control $(C O N)$ group. Original magnification: $6000 \times . M$, Mitochondria; $S L$, secondary lysosomes; $N$, nucleus; $L$, lipid droplets.

Because of the lack of specificity of the clinical signs and symptoms, adrenal gland injury might not be diagnosed; however, it often develops in patients in critical condition. A high incidence of relative adrenal failure (16\%-62\%) occurs in patients undergoing acute postoperative circulatory failure and requiring norepinephrine $(>0.2 \mu \mathrm{g} / \mathrm{kg} / \mathrm{min})$ during the 48 hours after cardiac surgery. ${ }^{25}$ Acute adrenal insufficiency is a life-threatening event that usually manifests when the adrenal cortex fails to produce sufficient glucocorticoids. ${ }^{26}$ Patients with adrenal insufficiency have worse cardiac function, need more inotropic support, and have higher lactate levels. ${ }^{27}$ However, excess corticosteroids might result in hyperglycemia and increased rates of infection, ${ }^{9,28}$ and excessive aldosterone promotes cardiac fibrosis and postoperative atrial fibrillation after cardiac surgery. ${ }^{29,30}$ In the present study, the CPB induced adrenal cortical morphological alterations and disturbed adrenocortical endocrine function. Hence, we should pay attention to the universality and harmfulness of adrenal injury.

This study showed marked cytological architectural and ultrastructural alterations in adrenocorticocytes in the CPB group, with cell mitochondria edema, ruptured mitochondrial cristae, leukocyte infiltration, and increased necrosis, apoptosis, and secondary lysosomes. The mitochondria, which play the most important role in steroidogenesis in the adrenal cortex, are the main cellular organelles affected and might affect postoperative steroidogenesis, potentially leading to adrenal insufficiency and an increase in serious complications. ${ }^{31}$ In addition, secondary lysosomes were significantly increased in the CPB group. Secondary lysosomes, important elements of autophagy, are responsible for cellular renovation and the degradation of intracytoplasmic materials, including injured organelles, proteins, and infectious agents. ${ }^{32,33}$ Autophagy might moderate inflammatory responses and protect against cell death. ${ }^{33}$ In the present study, increased secondary lysosomes might indicate CPB-induced adrenal injury and might protect against this injury via self-protection strategies.

The NLRP3 inflammasome is composed of an inflammasome sensor molecule, the adaptor protein apoptosisassociated speck-like protein containing C-terminal caspase recruitment domain and caspase 1. NLRP3 is triggered by pathogen-associated molecular patterns, damage-associated 

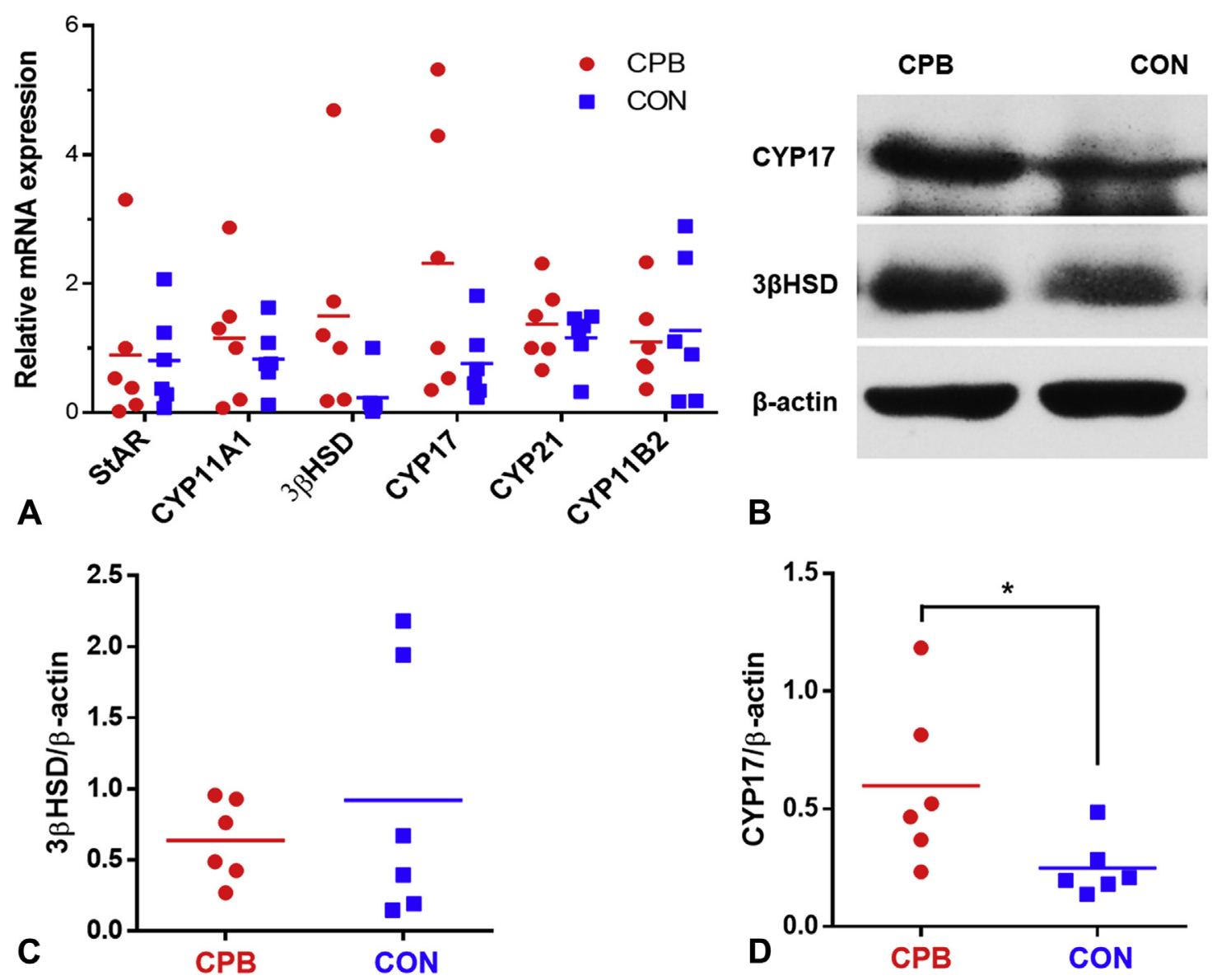

FIGURE 5. Steroidogenic enzymes in the adrenal glands. A, The mRNA expression levels of steroidogenic enzymes in the adrenal glands using real-time

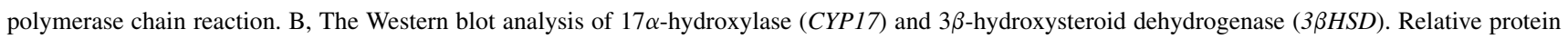
levels of CYP17 (C) and 3 $\beta \mathrm{HSD}$ (D) were determined after normalization with $\beta$-actin (compared with the control $[C O N]$ group, ${ }^{*} P<.05$ ). StAR, Steroidogenic acute regulatory protein; $C y P 11 A 1$, cytochrome $\mathrm{P} 450$ side chain cleavage; $C Y P 21,21$-hydroxylase; $C Y P 11 B 2$, aldosterone synthase; $C P B$, cardiopulmonary bypass.

molecular patterns, and bacterial toxins. ${ }^{12}$ NLRP3 activates caspase 1 , which proteolytically activates the proinflammatory cytokines IL-1 $\beta$ and IL-18. The NLRP3 inflammasome is involved in I/R injury and injurious inflammatory responses in various diseases. ${ }^{15,17-19,34}$ In the present study, lymphocyte and neutrophil infiltration was obvious in the CPB group. Activated lymphocytes and neutrophils secrete IL-1 $\beta$, IL-6, and TNF- $\alpha$ during the early inflammatory response. ${ }^{35}$ Furthermore, NLRP3 mRNA and NLRP3, and IL- $1 \beta$ proteins were highly expressed in the adrenal glands in the CPB group. Therefore, we hypothesize that this pathway might be involved in CPB-induced adrenal injury. Our previous experimental study showed that ellagic acid could prevent monocrotalininduced pulmonary artery hypertension and right ventricular hypertrophy via inhibiting NLRP3 inflammasome pathway activation. ${ }^{18}$ In addition, inhibition of NLRP3 inflammasome activation can limit inflammatory injury and reduce myocardial I/R injury. ${ }^{15,16}$ Therefore, we concluded that the NLRP3 inflammasome pathway might be a potential therapeutic target for CPB-induced adrenal injury.

\section{CONCLUSIONS}

In our model, CPB induces significant systemic and local inflammation in the adrenal tissue, resulting in cytological architectural and ultrastructural alterations in adrenocorticocytes. In addition, the NLRP3 inflammasome pathway might promote adrenal gland injury during $\mathrm{CPB}$, and might represent a novel potential therapeutic target. However, further studies are required to determine the mechanisms of the NLRP3 inflammasome pathway in adrenal gland injury during $\mathrm{CPB}$.

\section{Conflict of Interest Statement}

Authors have nothing to disclose with regard to commercial support. 


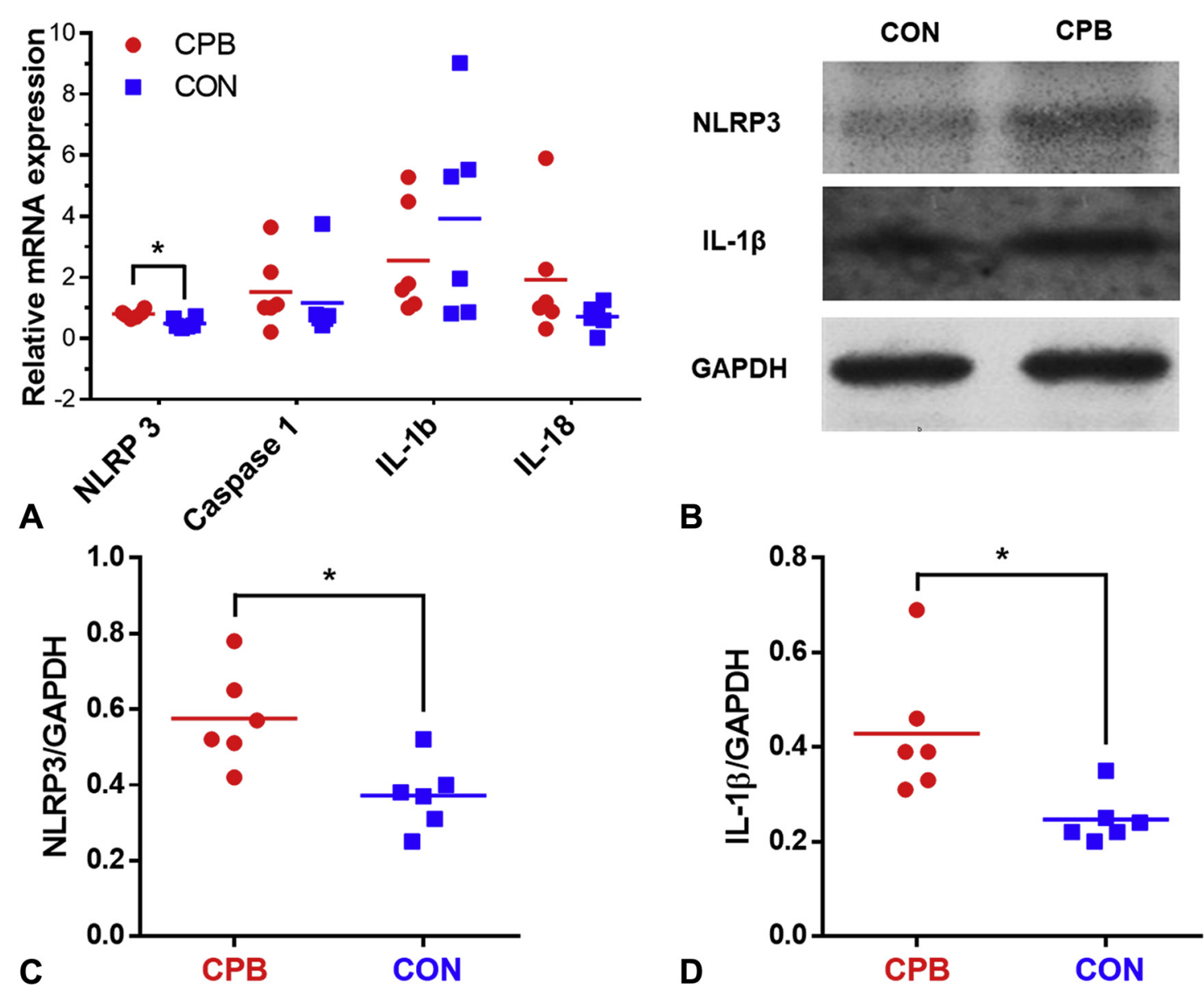

FIGURE 6. The nucleotide-binding oligomerization domain-like receptor containing pyrin domain 3 (NLRP3) inflammasome pathway. A, Adrenal mRNA expression levels of NLRP3, caspase 1, interleukin (IL)-1 $\beta$, and IL-18 using real-time polymerase chain reaction. B, Western blot analysis of NLRP3 and IL-1 $\beta$ were determined after normalization with glyceraldehyde-3-phosphate dehydrogenase (GAPDH) (compared with the control [CON] group, * $P<.05$ ). $C P B$, Cardiopulmonary bypass group.

The authors thank Dr Gang Dai (Key Laboratory of Health Ministry, Sun Yat-sen University, Guangzhou) for experimental assistance, Dr Wenshuang Ding (Department of Pathology, The First People's Hospital of Guangzhou, Guangzhou), and Yanyan Liu (Department of Pathology, The First Affiliated Hospital of Guangzhou University of Chinese Medicine, Guangzhou) for the tissue slides.

\section{References}

1. Whitlock RP, Devereaux PJ, Teoh KH, Lamy A, Vincent J, Pogue J, et al. Methylprednisolone in patients undergoing cardiopulmonary bypass (SIRS): a randomised, double-blind, placebo-controlled trial. Lancet. 2015;386:1243-53.

2. Hirao S, Minakata K, Masumoto H, Yamazaki K, Ikeda T, Minatoya K, et al. Recombinant human soluble thrombomodulin prevents acute lung injury in a rat cardiopulmonary bypass model. J Thorac Cardiovasc Surg. 2017;154:1973-83.

3. von Rhein M, Buchmann A, Hagmann C, Huber R, Klaver P, Knirsch W, et al. Brain volumes predict neurodevelopment in adolescents after surgery for congenital heart disease. Brain. 2014;137:268-76.

4. Belley-Cote EP, Parikh CR, Shortt CR, Coca SG, Garg AX, Eikelboom JW, et al. Association of cardiac biomarkers with acute kidney injury after cardiac surgery: a multicenter cohort study. J Thorac Cardiovasc Surg. 2016;152:245-51.

5. Adamik B, Kubler A, Gozdzik A, Gozdzik W. Prolonged cardiopulmonary bypass is a risk factor for intestinal ischaemic damage and endotoxaemia. Heart Lung Circ. 2017;26:717-23.
6. Ali-Hassan-Sayegh S, Mirhosseini SJ, Haddad F, Karimi-Bondarabadi AA, Shahidzadeh A, Weymann A, et al. Protective effects of corticosteroids in coronary artery bypass graft surgery alone or combined with valvular surgery: an updated and comprehensive meta-analysis and systematic review. Interact Cardiovasc Thorac Surg. 2015;20:825-36.

7. Jacob KA, Leaf DE, Dieleman JM, van Dijk D, Nierich AP, Rosseel PM, et al. Intraoperative high-dose dexamethasone and severe AKI after cardiac surgery. J Am Soc Nephrol. 2015;26:2947-51.

8. Scrascia G, Rotunno C, Guida P, Amorese L, Polieri D, Codazzi D, et al. Perioperative steroids administration in pediatric cardiac surgery: a meta-analysis of randomized controlled trials. Pediatr Crit Care Med. 2014;15:435-42.

9. Elhoff JJ, Chowdhury SM, Zyblewski SC, Atz AM, Bradley SM, Graham EM. Intraoperative steroid use and outcomes following the Norwood procedure: an analysis of the pediatric heart network's public database. Pediatr Crit Care Med. 2016;17:30-5.

10. Graham EM, Atz AM, McHugh KE, Butts RJ, Baker NL, Stroud RE, et al. Preoperative steroid treatment does not improve markers of inflammation after cardiac surgery in neonates: results from a randomized trial. J Thorac Cardiovasc Surg. 2014;147:902-8.

11. Kazmierski J, Kloszewska I. Is cortisol the key to the pathogenesis of delirium after coronary artery bypass graft surgery? Crit Care. 2011;15:102.

12. Strowig T, Henao-Mejia J, Elinav E, Flavell R. Inflammasomes in health and disease. Nature. 2012;481:278-86.

13. Yang G, Xue X, Chen Y, Song Z, Jiang Z, Hu K. Effects of cardiopulmonary bypass on lung nuclear factor-kappa B activity, cytokine release, and pulmonary function in dogs. Iran J Basic Med Sci. 2015;18:1233-9. 
14. Wang CT, Zhang L, Wu HW, Wei L, Xu B, Li DM. Doxycycline attenuates acute lung injury following cardiopulmonary bypass: involvement of matrix metalloproteinases. Int J Clin Exp Pathol. 2014;7:7460-8.

15. Toldo S, Marchetti C, Mauro AG, Chojnacki J, Mezzaroma E, Carbone S, et al. Inhibition of the NLRP3 inflammasome limits the inflammatory injury following myocardial ischemia-reperfusion in the mouse. Int J Cardiol. 2016:209:215-20.

16. Wang Y, Yan X, Mi S, Li Z, Wang Y, Zhu H, et al. Naoxintong attenuates ischaemia/reperfusion injury through inhibiting NLRP3 inflammasome activation. J Cell Mol Med. 2017;21:4-12.

17. Kuipers MT, Aslami H, Janczy JR, van der Sluijs KF, Vlaar AP, Wolthuis EK, et al. Ventilator-induced lung injury is mediated by the NLRP3 inflammasome. Anesthesiology. 2012;116:1104-15.

18. Tang B, Chen GX, Liang MY, Yao JP, Wu ZK. Ellagic acid prevents monocrotaline-induced pulmonary artery hypertension via inhibiting NLRP3 inflammasome activation in rats. Int J Cardiol. 2015;180:134-41.

19. Progatzky F, Sangha NJ, Yoshida N, McBrien M, Cheung J, Shia A, et al. Dietary cholesterol directly induces acute inflammasome-dependent intestinal inflammation. Nat Commun. 2014;5:5864.

20. Qin H, Chen GX, Liang MY, Rong J, Yao JP, Liu H, et al. The altered expression profile of microRNAs in cardiopulmonary bypass canine models and the effects of mir-499 on myocardial ischemic reperfusion injury. J Transl Med. 2013;11:154.

21. Rong J, Ye S, Wu ZK, Chen GX, Liang MY, Liu H, et al. Controlled oxygen reperfusion protects the lung against early ischemia-reperfusion injury in cardiopulmonary bypasses by downregulating high mobility group box 1. Exp Lung Res. 2012;38:183-91.

22. Heckmann M, d'Uscio CH, Steckel H, Neuhaeuser C, Bodeker RH, Thul J, et al. Reduction in cortisol inactivation is part of the adrenal stress response to cardiac and noncardiac pediatric surgery: a prospective study using gas chromatography-mass spectrometry analysis. Horm Metab Res. 2014;46: 677-84.

23. Nasr DA, Abdelhamid HM. The efficacy of caudal dexmedetomidine on stress response and postoperative pain in pediatric cardiac surgery. Ann Card Anaesth. 2013;16:109-14.

24. Ngarmukos C, Grekin RJ. Nontraditional aspects of aldosterone physiology. Am J Physiol Endocrinol Metab. 2001;281:E1122-7.

25. Seravalli L, Pralong F, Revelly JP, Que YA, Chollet M, Chiolero R. Adrenal function after induction of cardiac surgery patients with an etomidate bolus: a retrospective study. Ann Fr Anesth Reanim. 2009;28:743-7.
26. Soto-Rivera CL, Agus MS, Sawyer JE, Macrae DJ. Pediatric Cardiac Intensive Care Society 2014 consensus statement: pharmacotherapies in cardiac critical care hormone replacement therapy. Pediatr Crit Care Med. 2016; 17:S59-68.

27. Ando M, Park IS, Wada N, Takahashi Y. Steroid supplementation: a legitimate pharmacotherapy after neonatal open heart surgery. Ann Thorac Surg. 2005;80: 1672-8.

28. Dreher M, Glatz AC, Kennedy A, Rosenthal T, Gaynor JW. A single-center analysis of methylprednisolone use during pediatric cardiopulmonary bypass. J Extra Corpor Technol. 2015;47:155-9.

29. Alexandre J, Saloux E, Chequel M, Allouche S, Ollitrault P, Plane AF, et al. Preoperative plasma aldosterone and the risk of atrial fibrillation after coronary artery bypass surgery: a prospective cohort study. J Hypertens. 2016; 34:2449-57.

30. Chequel M, Ollitrault P, Saloux E, Parienti JJ, Fischer MO, Desgue J, et al. Preoperative plasma aldosterone levels and postoperative atrial fibrillation occurrence following cardiac surgery: a review of literature and design of the ALDO-POAF study (ALDOsterone for prediction of post-operative atrial fibrillation). Curr Clin Pharmacol. 2016;11:150-8.

31. Chen KT, Lin TY, Foo NP, Lin HJ, Guo HR. Traumatic adrenal haematoma: a condition rarely recognised in the emergency department. Injury. 2007:38:584-7.

32. Mizushima N, Komatsu M. Autophagy: renovation of cells and tissues. Cell. 2011;147:728-41.

33. Rubinsztein DC, Bento CF, Deretic V. Therapeutic targeting of autophagy in neurodegenerative and infectious diseases. J Exp Med. 2015;212: 979-90.

34. Wu D, Ren P, Zheng Y, Zhang L, Xu G, Xie W, et al. NLRP3 (nucleotide oligomerization domain-like receptor family, pyrin domain containing 3)-caspase-1 inflammasome degrades contractile proteins: implications for aortic biomechanical dysfunction and aneurysm and dissection formation. Arterioscler Thromb Vasc Biol. 2017;37:694-706.

35. Agoro R, Piotet-Morin J, Palomo J, Michaudel C, Vigne S, Maillet I, et al. IL1R1-MyD88 axis elicits papain-induced lung inflammation. Eur J Immunol 2016;46:2531-41

Key Words: cardiopulmonary bypass, adrenal injury, NLRP3, cardiac surgery 